\title{
Metacognición en el Aprendizaje de la Anatomía
}

\author{
Metacognition in the Learning of Anatomy
}

Oscar Andrés Alzate Mejía ${ }^{1,2}$ \& Oscar Eugenio Tamayo Alzate ${ }^{2}$

\begin{abstract}
ALZATE, M. O. A. \& TAMAYO, A. O. E. Metacognición en el aprendizaje de la anatomía. Int. J. Morphol., 37(1):7-11, 2019.
RESUMEN: Actualmente la enseñanza y aprendizaje de la Anatomía tiene grandes retos que debe superar: los estudiantes están abrumados por gran cantidad de información; generalmente el aprendizaje está basado en la memoria; la enseñanza esta carente de métodos que permitan hacer consciente el aprendizaje; falta mejorar estrategias evaluativas que demuestren aprendizajes. Por su parte, la metacognición, entendida como el dominio que tiene el sujeto sobre sus propios procesos cognoscitivos busca propiciar en los estudiantes la regulación de sus propios aprendizajes. Este trabajo describe el aporte de la metacognición al aprendizaje de la Anatomía. Esta investigación es de tipo cualitativo. Fue un estudio de caso que analizó cómo un estudiante incorporó la metacognición al aprendizaje de la Anatomía. En el desarrollo del trabajo se indagaron obstáculos de aprendizaje, se diseñaron, aplicaron y analizaron diferentes actividades sobre aprendizaje metacognitivo de la Anatomía. Se incorporó la metacognición al aprendizaje de la Anatomía encontrando que la planeación hace el estudio más organizado, provocando mayor consciencia de cada actividad de aprendizaje y permitiendo obtener mejores resultados. El monitoreo halló que la mayor dificultad en el aprendizaje de la Anatomía se encuentra en la capacidad de orientación espacial. Finalmente la evaluación consideró el progreso en el aprendizaje de la Anatomía justificado en el control del tiempo, en un nuevo lenguaje con términos más adecuados, en la seguridad y confianza frente a las evaluaciones.
\end{abstract}

PALABRAS CLAVE: Didáctica de la Anatomía; Metacognición; Enseñanza, Aprendizaje.

\section{INTRODUCTION}

Actualmente el mundo necesita profesionales de la salud con una amplia formación académica, personal y con la capacidad de realizar actividades que prevengan la enfermedad y promuevan la salud. Es fundamental educar personas competentes desde el campo científico, tecnológico y humano; urge formar profesionales que apliquen su conocer, entre otras, al saber hacer. Para el efecto, las ciencias básicas biomédicas son imprescindibles en la formación de los profesionales de la salud (Grande, 2009).

La Anatomía, como la ciencia que estudia las estructuras donde ocurren las funciones vitales del cuerpo humano (Moore et al., 2014) es el eje que moviliza las ciencias biomédicas. La Anatomía es pilar esencial; acerca directamente el estudiante al ser humano, le permite reconocer las estructuras donde ocurren las funciones vitales más maravillosas del cuerpo humano y es fundamental para entender su funcionamiento y las patologías. La Anatomía constituye la base de la práctica de las profesiones de salud, permite realizar la exploración física y utilizar las técnicas más modernas de obtención de imágenes.
En conclusión, la capacidad para interpretar una observación clínica correctamente es el punto final de una comprensión anatómica profunda (Drake \& Vogle, 2010).

Sin embargo, la situación actual de la enseñanza y aprendizaje de la Anatomía y de las demás ciencias básicas biomédicas, tiene grandes retos que debe superar: la desintegración entre las ciencias básicas y las ciencias clínicas; un enfoque centrado en la enseñanza, más que en el aprendizaje; resultados bajos en el aprendizaje y existe insuficiente disponibilidad de profesores con formación en el área (Triana Contreras, 2013). Estos problemas han dado lugar a programas recargados. Los estudiantes de Anatomía (que por lo general cursan primeros semestres y que se están habituando a las exigencias de la vida universitaria, a hábitos de estudio y de lectura), están abrumados por la gran cantidad de detalles e información. Generalmente el aprendizaje está basado en una educación memorística; la principal finalidad es aprobar exámenes. Los amplios contenidos y las horas dispuestas para clases han hecho que la gran cantidad de información no se pro-

\footnotetext{
${ }^{1}$ Departamento de Ciencias Básicas de la Universidad Autónoma de Manizales, Manizales, Colombia.

${ }^{2}$ Departamento de Estudios Educativos, Universidad de Caldas, Manizales, Colombia.
} 
cese ni se convierta en un aprendizaje significativo que pueda aplicarse. La enseñanza de la Anatomía esta carente de métodos adecuados que permitan al estudiante pensar y hacerse consciente de su aprendizaje, falta implementar y mejorar estrategias evaluativas que demuestren aprendizajes profundos en los estudiantes y falta promover el aprendizaje práctico en donde el estudiante disfrute del laboratorio al manipular las estructuras anatómicas (Korf et al., 2008). Desde lo anterior, el propósito de este trabajo es describir el aporte de la metacognición a los procesos de aprendizaje de la Anatomía. La metacognición, entendida como el dominio y regulación que tiene el sujeto sobre sus propios procesos cognoscitivos (Flavell, 1979) busca propiciar en los estudiantes de Anatomía la regulación de sus propios aprendizajes, orientándolos a ser aprendices autónomos. Se espera que una enseñanza y aprendizaje metacognitivos en Anatomía aporten a la formación de la agudeza perceptiva y la capacidad de cuestionar analíticamente; aporte a la formación de personas críticas, autónomas, pensantes, productivas, con habilidades para continuar aprendiendo y con una actitud de actualización permanente; se espera que estos estudiantes tengan la capacidad de valorar alternativas, estar bien informados, desarrollar y defender posiciones, formular preguntas apropiadas y derivar conclusiones justificadas. Aprendizajes profundos de la Anatomía son necesarios para todos los futuros profesionales de la salud, una Anatomía bien aprendida tendrá como fruto una excelente carrera académica y un exitoso desempeño profesional.

\section{MATERIAL Y MÉTODO}

Esta investigación es un estudio de caso único con el cual se hizo un análisis en profundidad para entender como un estudiante incorporó la metacognición al aprendizaje de la Anatomía. Se hace un reporte de éste caso. La selección se hizo desde estudiantes de primer semestre de Anatomía de Fisioterapia de la Universidad Autónoma de Manizales en el primer semestre de 2014. Para la selección se leyeron las respuestas de los 40 estudiantes que participaron, de estos se escogieron las 62 respuestas de la joven en las que se basó la investigación. Los criterios de selección que se tuvieron en cuenta fueron: tiempo invertido para responder completamente a las preguntas; calidad y fluidez de las respuestas; cantidad de la información suministrada; interés y participación. A futuro se espera ampliar la investigación con el total de los estudiantes.

Se esperó que un adecuado actuar metacognitivo del profesor produjera aprendizajes metacognitivos en los estudiantes. Se diseñaron y ejecutaron actividades de apren- dizaje donde se incluyeron diferentes acciones metacognitivas. Después se analizó el cambio en el aprendizaje de la estudiante y cómo esas actividades de aula pudieron incorporar la metacognición. Se incluyeron actividades como son: la planeación, el monitoreo y la evaluación. Finalmente el conjunto de actividades de enseñanza, su reflexión y el conjunto de actividades de aprendizaje con su reflexión constituyeron una Guía de aprendizaje metacognitivo de la Anatomía.

La presente investigación se desarrolló en cinco fases o momentos específicos:

\section{Revisión teórica e indagación sobre obstáculos de apren-} dizaje. Se revisó bibliografía sobre la enseñanza de la Anatomía, su aprendizaje y la Metacognición (Naug et al., 2011). Se enfatizó la revisión en el aprendizaje metacognitivo. Después se indagó sobre los obstáculos en la didáctica de la Anatomía. Para ello se encuestó a 5 docentes del área de las Universidades Autónoma, Caldas y Manizales, también a monitores de la asignatura y se entrevistó a los mismos estudiantes sobre las dificultades que presentaban al momento de enseñar y aprender la Anatomía.

II. Diseño de actividades sobre aprendizaje metacognitivo de la Anatomía. Se diseñaron y validaron un total de ocho instrumentos que indagaron a los estudiantes con 62 preguntas que incluyeron la dimensión afectiva en Anatomía, ideas previas de miembro superior e interrogantes de reflexión metacognitiva en el aprendizaje. Estos instrumentos se diseñaron y aplicaron a la par con una guía de estudio que es una serie de actividades de aprendizaje con imágenes vídeos, estructuras biológicas y radiografías. Estas actividades se hicieron con lecturas temáticas, dibujos, modelados con plastilina, estudios multimediales y visitas al laboratorio con estructuras anatómicas. Actualmente las guías de estudio se encuentran disponibles virtualmente para que los estudiantes de pregrado y de posgrado las puedan consultar y descargar constantemente. Se puede tener acceso a ellas en este momento en: http:// fisiolofar.blogspot.com/ (Alzate Mejía, 2013).

III.Aplicación de las actividades de aprendizaje metacognitivo de la Anatomía. Durante el primer semestre de 2014 y después de que a los estudiantes se les había orientado una introducción a la metacognición, se les orientó la unidad de Miembro superior en la cual se incorporaron las actividades de aprendizaje metacognitivo de la Anatomía. La Anatomía músculo esquelética de miembro superior se estudió en ese momento después de fundamentos de Anatomía, Dorso, tórax y miembro inferior. Se desarrolló en 4 semanas y media para un total de 9 clases, 4 asesorías y 4 monitorias. 
IV. Recolección de la información. Por medio de las respuestas a los diferentes instrumentos, las actividades de la guía de estudio, fotografías y videos se recolectó la información que mostró la incorporación de la metacognición en el aprendizaje de la Anatomía.

V. Análisis de la información. Se analizó e interpretó la información desde las 62 diferentes respuestas que dio la estudiante (estudio de caso) a los 8 instrumentos de investigación. Además de evidenciar obstáculos de aprendizaje e ideas previas, se organizaron las respuestas en 6 diferentes categorías a saber: Afectividad, Aprendizaje práctico virtual, Aprendizaje práctico con el laboratorio, Planeación, Monitoreo, Evaluación. Seguidamente y desde las respuestas de la estudiante se seleccionaron las subcategorías que se repetían más en cada una de las categorías, encontrando la relación de los hallazgos con el marco teórico y la cualificación del aprendizaje de la Anatomía por medio de la Metacognición.

\section{RESULTADOS Y DISCUSIÓN}

Obstáculos de aprendizaje en la didáctica de la Anatomía: para iniciar las actividades que orientarían a los estudiantes, se obtuvieron las diferentes dificultades en la didáctica de la Anatomía. Los principales obstáculos desde las respuestas de docentes, monitores y estudiantes fueron los siguientes:

- Carencia de estrategias para aprovechar la memoria. Los estudiantes llegan a la Universidad sin actividades eficientes que le hayan permitido un buen desarrollo de la memoria (Triana Contreras). Ellos piensan que esta habilidad no es útil para la enseñanza, al contrario los docentes podrían caer en el error que solo es importante la memoria en el aprendizaje de la Anatomía (Alterio Ariola \& Ruiz Bolívar, 2010).

- Poco desarrollo de la habilidad visoespacial. Una de las mayores dificultades que dicen los estudiantes poseer a la hora de aprender Anatomía es su poca "capacidad de ubicación". La enseñanza de la Anatomía descuida estos aspectos importantes y tampoco tiene habitualmente actividades previas que ayuden a la evaluación y desarrollo de la habilidad visoespacial (Korf et al.; Hegarty et al., 2009; Vorstenbosch et al., 2013; Sweeney et al., 2014).

- Pocas prácticas en laboratorios virtuales, con modelos y con estructuras biológicas. Si bien es cierto que existen dificultades con la enseñanza de la Anatomía a través de estructuras biológicas, las horas destinadas a las prácticas son insuficientes para abarcar los contenidos (Triana Contreras). Algunas clases de Anatomía se orientan más hacia la teoría, no se da la importancia requerida a las prácticas con análisis de imágenes, por medio de estrategias virtuales y menos con estructuras biológicas (Guiraldes et al., 2001).

- Enseñanza que relaciona poco las estructuras anatómicas entre sí mismas, la función y la enfermedad. Generalmente la enseñanza de la Anatomía se hace de una manera memorística, donde se le presenta a los estudiantes una serie de estructuras del cuerpo y su respectiva ubicación sin que esto tenga un sentido con la profesión de los estudiantes.

- Falta de dedicación y métodos adecuados de aprendizaje. En la enseñanza de la Anatomía, lo más fácil podría ser justificar el poco progreso del aprendizaje de los estudiantes en su falta de dedicación y en métodos inapropiados. Pero como se ha visto, los obstáculos de aprendizaje no solo se centran en la falta de estudio y en sus formas de aprender (Triana Contreras).

A continuación se presentará el análisis de las 6 categorías diferentes del estudio desde las respuestas dadas por la estudiante del caso:

Afectividad en la didáctica de la Anatomía. En las respuestas mostradas por la estudiante se pudo observar que existe satisfacción al estudiar Anatomía. Se encuentran en este nivel con el cuerpo humano, con la terminología propia de su profesión y con el reconocimiento de las estructuras del cuerpo. De la misma manera, las respuestas, permitieron demostrar el aporte vocacional que hace la Anatomía a la estudiante, esta asignatura le permite a los estudiantes visualizarsu profesión, le permitirá enfrentarse al reconocimiento de estructuras de su futuro paciente y le mostrará el rigor en el estudio.

Aprendizaje práctico virtual. En el estudio de la Anatomía las imágenes son fundamentales para el aprendizaje (Johnson et al., 2012), En las respuestas se pudo evidenciar el aporte que realiza la enseñanza virtual al aprendizaje de la Anatomía a la estudiante (Guiraldes et al.), permitió entre otros reconocer las articulaciones entre los huesos, el origen, inserción y visualización de algunos músculos. Un aspecto fundamental de los vídeos e imágenes es que ayuda a la estudiante con su ubicación. Por otro lado, los videos y otras ayudas virtuales que se encuentran en el blog http://fisiolofar.blogspot.com/ (Alzate Mejía, 2013; Jaffar, 2012) le aportaron al aprendizaje de detalles óseos pero no mucho para la miología, para los músculos se le hizo más fácil con réplicas y estructuras biológicas. 
Entonces le parece que sería ideal que los vídeos fueran con estructuras reales para ser más consciente de la estructura y tener una mayor claridad (Duque Parra \& Barco Ríos, 2013).

Aprendizaje práctico con el laboratorio. En las respuestas sobre la categoría aprendizaje con laboratorios se considera la valiosa importancia del estudio práctico de la Anatomía. Por un lado motiva al estudiante en su profesión, lo acerca de cierta manera con pacientes reales en donde en algún momento hubo vida. Así mismo lo motiva por la relación que tienen todos los estudiantes de las facultades de salud con los cadáveres, le hace cobrar identidad por su área y su profesión (Inzunza et al., 2003). Estudiar Anatomía con estructuras biológicas hace que los estudiantes comprendan más fácil la teoría. El hecho de ver y sobre todo palpar hacen que la enseñanza se facilite y el aprendizaje sea con mayor profundidad. La manipulación de las estructuras hace que ellos confirmen la teoría después de leer los textos, ver imágenes e ilustrarse con vídeos (Saltarelli et al., 2014). Además de todo, permite la interacción con otros estudiantes al dialogar sobre las estructuras a la luz teórica y las fotografías de atlas. Sin embargo, la estructura por sí sola no podría tener utilidad, se necesita la relación con otras estructuras de los mismos cadáveres, la funcionalidad de cada una y la relación con diferentes patologías (Montemayor Flores, 2006).

Planeación. De acuerdo con las respuestas anteriores la planeación, que implica la selección de estrategias apropiadas, aporta favorablemente al aprendizaje de la Anatomía. Ésta hace el estudio sea más organizado provocando más consciencia en cada actividad de aprendizaje, de la misma manera permitió en la estudiante obtener mejores resultados.

La planeación en los estudiantes de Anatomía aporta de manera fundamental a su aprendizaje. Pero esta planeación debe estar unida a la planeación de la enseñanza. Una buena planeación en la enseñanza y aprendizaje de la Anatomía, sustentada desde las respuestas de la estudiante, debería contar con acciones diferentes en un orden que no necesariamente debe ser común a todos. Esta planeación de acuerdo a esto debería incluir una lectura del tema con el análisis de las imágenes que ofrecen los buenos textos, se hace necesario que el docente de las indicaciones respectivas acerca de los temas que se debe enfatizar. Esa lectura sería ideal que fuera activa, aquí sería fundamental que el estudiante realice dibujos de las estructuras y tablas que expliquen los músculos, los nervios y vasos. Después de esta primera lectura o, antes si es la estrategia del estudiante, se debe dar la posibilidad del aprendizaje virtual, es decir ver un vídeo o utilizar aplicaciones virtuales que aclaren las lecturas. A continuación, el estudiante puede aprender manipulando estructuras biológicas acompañado de un Atlas. Esta actividad sería ideal que la pudiera realizar con otro estudiante con el cual pueda generar discusión. Seguidamente el estudiante puede asistir con el docente para resolver dudas, profundizar la temática, evaluar lo estudiado, aclarar la identificación de estructuras, sus funciones y la relación con otras estructuras y diversas patologías. Finalmente, el estudiante puede llegar a otra lectura que puede ser directa del texto, de lo que subrayó o resumió e idealmente con otros estudiantes puede asistir nuevamente al laboratorio para complementar sus enseñanzas, esta visita puede ser acompañada por la tutoría de su docente o por estudiantes monitores si es su necesidad.

Monitoreo. El monitoreo que permite la posibilidad de comprender y modificar la ejecución de la tarea para así verificar, rectificar y revisar las estrategias seguidas (Martí, 1995), demostró desde las respuestas de la estudiante que la mayor dificultad en el aprendizaje de la Anatomía se encuentra en la capacidad de orientación espacial. Esta necesidad se presenta al ubicar estructuras como huesos y músculos al igual que en radiografías. Para dar solución a ésta dificultad, las respuestas muestran que la estudiante hizo actividades tales como lectura de textos de Anatomía la descripción sobre la orientación, ver los vídeos propuestos, estudiar en compañía, seguir las indicaciones de la Unidad didáctica y principalmente utilizó las estructuras del laboratorio.

Evaluación. Finalmente, desde las respuestas concernientes a la categoría evaluación, la estudiante considera progreso en su aprendizaje de la Anatomía. Dos aspectos relevantes Justifican este avance. Por un lado siente que controla más su tiempo para lograr mejores resultados (Angulo Delgado \& García Rovira, 2009). Por otro lado siente mayor seguridad y confianza frente a las evaluaciones tanto prácticas como teóricas. El manejo del tiempo es un aspecto importante ya que la estudiante valora los resultados de las estrategias seguidas en términos de eficacia (Martí); ella tuvo suficiente con el desarrollo de su planeación, fue importante para ella el hecho de no tener que invertir tiempo reforzando sobre lo estudiado, y alcanza su compresión con mayor rapidez.

En general, la incorporación de la metacognición permitió a la estudiante reconocer el éxito en su proceso de aprender (Monereo, 1995), le mejoró el rendimiento académico (Turan \& Demirel, 2010), le aportó aprendizajes en profundidad y motivación hacia la profesión en formación (Escanero-Marcén et al., 2013). 
ALZATE, M. O. A. \& TAMAYO, A. O. E. Metacognition in the learning of anatomy. Int. J. Morphol., 37(1):7-11, 2019.

SUMMARY: Currently there are important challenges in the teaching and learning of Anatomy. Students are overwhelmed by all of the information and generally, learning is based on memory. Furthermore, teaching instruction lacks methods that encourage cognitive learning. It is necessary to improve evaluation strategies that demonstrate learning. Metacognition, is understood as the command an individual has over their own cognitive processes aims to encourage students to regulate their own learning process. This paper describes the contribution of metacognition to Anatomy learning. This research is qualitative; it was a case study that analyzed how a student incorporated metacognition into Anatomy learning. During the development of this study, learning obstacles were investigated, different activities on metacognitive learning of the Anatomy were designed, applied and analyzed. The research incorporated metacognition into the learning of Anatomy and determined that planning allowed for better study organization, created greater awareness of each learning activity and showed better results. During monitoring, it was found that the greatest difficulty in learning Anatomy, was understanding spatial awareness. Finally the evaluation considered the learning progress of anatomy based on time control, new language, more adequate terms, and greater assurance and confidence during the evaluations.

KEY WORDS: Anatomy Didactics; Metacognition; Teaching; Learning.

\section{REFERENCIAS BIBLIOGRÁFICAS}

Alterio Ariola, G. H. \& Ruiz Bolívar, C. Mediación metacognitiva, estrategias de enseñanza y procesos de pensamiento del docente de Medicina. Educ. Med. Super., 24(1):25-32, 2010.

Alzate Mejía, O. A. Incorporación de blog y redes sociales a la enseñanza y aprendizaje de la morfofisiología. Rev. Med. Risaralda, 19(Supl. 2):278, 2013.

Angulo Delgado, F. \& García Rovira, P. Aprender a enseñar ciencias: una propuesta basada en la autorregulación. Rev. Educ. Pedagog., (25):6786, 2009.

Drake, R. \& Vogle, W. Gray. Anatomía para Estudiantes. $2^{\mathrm{a}}$ ed. Barcelona, Elsevier, 2010.

Duque Parra, J. E. \& Barco Ríos, J. Enseñanza de la ciencia sin experimentación por demostración versus enseñanza por virtualización de la experimentación. Arch. Med. (Manizales), 13(2):226-32, 2013.

Escanero-Marcén, J. F.; Soria, M. S.; Escanero-Ereza, M. E. \& GuerraSánchez, M. Influencia de los estilos de aprendizaje y la metacognición en el rendimiento académico de los estudiantes de fisiología. Rev. Fund. Educ. Med., 16(1):23-9, 2013.

Flavell, J. H. Metacognition and cognitive monitoring: A new area of cognitive-developmental inquiry. Am. Psychol., 34(10):906-11, 1979.

Grande, J. P. Training of physicians for the twenty-first century: role of the basic sciences. Med. Teach., 31(9):802-6, 2009.

Guiraldes, H.; Oddó, H.; Mena, B.; Velasco, N. \& Paulos, J. Teaching human anatomy: experience and challenge in a medical school. Rev. Chil. Anat., 19(2):205-12, 2001.

Hegarty, M.; Keehner, M.; Khooshabeh, P. \& Montello, D. R. How spatial abilities enhance, and are enhanced by, dental education. Learn. Individ. Differ, 19(1):61-70, 2009.
Inzunza, O.; D’Acuña, E. \& Bravo, H. Practical evaluation in anatomy. Performance of first year medical students in relation to different ways of asking questions. Int. J. Morphol., 21(2):131-6, 2003.

Jaffar, A. A. YouTube: An emerging tool in anatomy education. Anat. Sci. Educ., 5(3):158-64, 2012.

Johnson, E. O.; Charchanti, A. V. \& Troupis, T. G. Modernization of an anatomy class: From conceptualization to implementation. A case for integrated multimodal-multidisciplinary teaching. Anat. Sci. Educ., 5(6):354-66, 2012.

Korf, H. W.; Wicht, H.; Snipes, R. L.; Timmermans, J. P.; Paulsen, F.; Rune, G. \& Baumgart-Vogt, E. The dissection course - necessary and indispensable for teaching anatomy to medical students. Ann. Anat., 190(1):16-22, 2008.

Martí, E. Metacognición: Entre la fascinación y el desencanto. Infancia Aprendiz., 72:9-32, 1995.

Monereo, C. Enseñar a conciencia ¿Hacia una didáctica metacognitiva?. Aula Innov. Educ., (34):74-80, 1995.

Montemayor Flores, B. G. What the dissection meaning for medical students. Int. J. Morphol., 24(4):575-80, 2006.

Moore, K.; Dalley, A. \& Agur, A. Anatomía con Orientación Clínica. $7^{\mathrm{a}}$ ed. Barcelona, Kluwer Wolters, 2014.

Naug, H. L.; Colson, N. J. \& Donner, D. G. Promoting metacognition in first year anatomy laboratories using plasticine modeling and drawing activities: a pilot study of the "blank page" technique. Anat. Sci. Educ., 4(4):231-4, 2011.

Saltarelli, A. J.; Roseth, C. J. \& Saltarelli, W. A. Human cadavers Vs. multimedia simulation: A study of student learning in anatomy. Anat. Sci. Educ., 7(5):331-9, 2014.

Sweeney, K.; Hayes, J. A. \& Chiavaroli, N. Does spatial ability help the learning of anatomy in a biomedical science course? Anat. Sci. Educ., 7(4):289-94, 2014.

Triana Contreras, Z. M. La Enseñanza de las Ciencias Básicas Médicas. Nuevo León, Universidad de Monterrey, 2013.

Turan, S. \& Demirel, Ö. In what level and how medical students use metacognition? A case from Hacettepe University. Procedia Soc. Behav. Sci., 2(2):948-52, 2010.

Vorstenbosch, M. A.; Klaassen, T. P.; Donders, A. R.; Kooloos, J. G.; Bolhuis, S. M. \& Laan, R. F. Learning anatomy enhances spatial ability. Anat. Sci. Educ., 6(4):257-62, 2013.

Dirección para correspondencia:

Oscar Andrés Alzate Mejía

Departamentos de Ciencias Básicas

Universidad Autónoma de Manizales

Manizales

COLOMBIA

Email: oalzate@autonoma.edu.co

Recibido : 09-02-2018

Aceptado : 27-09-2018 